\title{
First Detection of a Strong Magnetic Field on a Bursty Brown Dwarf: Puzzle Solved
}

\author{
S. V. Berdyugina ${ }^{1,2}$, D. M. Harrington ${ }^{1,2}$, O. Kuzmychov ${ }^{1}$ (i) , J. R. Kuhn ${ }^{2}$, G. Hallinan ${ }^{3}$, A. F. Kowalski ${ }^{4,5}$, and S. L. Hawley ${ }^{6}$ (1) \\ ${ }^{1}$ Kiepenheuer Institut für Sonnenphysik, Schöneckstrasse 6, D-79104 Freiburg, Germany; sveta@leibniz-kis.de \\ 2 Institute for Astronomy, University of Hawaii, 2680 Woodlawn Drive, Honolulu, 96822-1897 HI, USA \\ ${ }^{3}$ California Institute of Technology, 1200 East California Boulevard, Pasadena, CA 91125, USA \\ ${ }^{4}$ Department of Astrophysical and Planetary Sciences, University of Colorado Boulder, 2000 Colorado Avenue, Boulder, CO 80305, USA \\ ${ }^{5}$ National Solar Observatory, University of Colorado Boulder, 3665 Discovery Drive, Boulder, CO 80303, USA \\ ${ }^{6}$ Department of Astronomy, University of Washington, 15th Avenue NE, Seattle, WA 98195, USA \\ Received 2016 February 12; revised 2017 August 10; accepted 2017 August 11; published 2017 September 20
}

\begin{abstract}
We report the first direct detection of a strong, $5 \mathrm{kG}$ magnetic field on the surface of an active brown dwarf. LSR $\mathrm{J} 1835+3259$ is an M8.5 dwarf exhibiting transient radio and optical emission bursts modulated by fast rotation. We have detected the surface magnetic field as circularly polarized signatures in the $819 \mathrm{~nm}$ sodium lines when an active emission region faced the Earth. Modeling Stokes profiles of these lines reveals the effective temperature of $2800 \mathrm{~K}$ and $\log$ gravity acceleration of 4.5 . These parameters place LSR J1835+3259 on evolutionary tracks as a young brown dwarf with the mass of $55 \pm 4 M_{\mathrm{J}}$ and age of $22 \pm 4 \mathrm{Myr}$. Its magnetic field is at least $5.1 \mathrm{kG}$ and covers at least $11 \%$ of the visible hemisphere. The active region topology recovered using line profile inversions comprises hot plasma loops with a vertical stratification of optical and radio emission sources. These loops rotate with the dwarf in and out of view causing periodic emission bursts. The magnetic field is detected at the base of the loops. This is the first time that we can quantitatively associate brown dwarf non-thermal bursts with a strong, $5 \mathrm{kG}$ surface magnetic field and solve the puzzle of their driving mechanism. This is also the coolest known dwarf with such a strong surface magnetic field. The young age of LSR J1835+3259 implies that it may still maintain a disk, which may facilitate bursts via magnetospheric accretion, like in higher-mass T Tau-type stars. Our results pave a path toward magnetic studies of brown dwarfs and hot Jupiters.
\end{abstract}

Key words: brown dwarfs - magnetic fields - polarization - stars: individual (LSR J1835+3259)

\section{Introduction}

Brown dwarfs are intermediate between stars and planets. Because of their low masses, they are incapable of hydrogen fusion in the core, so they radiate energy due to deuterium burning, slow gravitational collapse, and thermal cooling. There is growing evidence that brown dwarfs may possess rather strong magnetic fields, similar to active, early M-type red dwarf stars of larger masses and higher temperatures (JohnsKrull \& Valenti 2000). It was found that non-radiative heating of plasma, evidenced by chromospheric hydrogen emission and coronal X-ray radiation, sharply decline in brown dwarfs (Neuhäuser et al. 1999; Mohanty \& Basri 2003; Preibisch et al. 2005; Grosso et al. 2007). Whether this reduction implies a drop in the surface magnetic field strength remains a puzzle.

One clue comes from extremely energetic flares that are detected in UV and X-ray radiation as well as in the optical hydrogen Balmer emission, providing an indication for magnetic reconnection events (Schmidt et al. 2007). Other evidence for magnetic activity in ultra-cool dwarfs is the presence of both quiescent and flaring non-thermal radio emission (e.g., Berger et al. 2001; Berger 2006; Hallinan et al. 2006, 2007; Antonova et al. 2007). A number of them exhibit transient events with periodic radio bursts and aurora-like, hydrogen and metal line optical emission (Hallinan et al. 2007, 2015) but no X-ray corona as in warmer, more massive, active red dwarf stars (Neuhäuser et al. 1999; Preibisch et al. 2005; Grosso et al. 2007). The radio luminosity of some ultracool $\mathrm{M}$ dwarfs and brown dwarfs is even higher than that of warmer, earlier-type active $\mathrm{M}$ dwarfs, and it continues to increase beyond spectral type M8 (Berger 2006), the border where both cool, old low-mass stars and warm, young brown dwarfs can be found. This peculiar behavior suggests that the classical hot stellar chromosphere and corona change dramatically across this mass range.

Several very-low-mass ultra-cool stars and brown dwarfs (classes M8 to T6), showing quiet non-thermal radio emission were found to be sources of transient but periodic and highly circularly polarized bursts at a few $\mathrm{GHz}$, with periods of $2-3 \mathrm{hr}$, which were associated with rotational periods of dwarfs (Berger et al. 2005; Hallinan et al. 2006, 2007). It was suggested (Hallinan et al. 2006) that these bursts may be produced at polar regions of a large-scale magnetic field, by a coherent process such as the electron cyclotron maser (ECM) instability. This mechanism is known to be responsible for the radio emission at $\mathrm{kHz}$ and $\mathrm{MHz}$ frequencies from the magnetized planets (e.g., Jupiter) in our solar system. If the same mechanism is responsible for radio bursts in ultra-cool dwarfs at frequencies of few $\mathrm{GHz}$, the magnetic field of a few kilo-Gauss is required on the dwarf surface (Hallinan et al. 2006), i.e., as high as those possessed by earlier-type classical M-type flare stars. There is also evidence that the radio pulses correlate with bright regions seen in the optical light curves (Harding et al. 2013; Hallinan et al. 2015). The question of whether these bright spots are magnetic, as some models suggest (Kuznetsov et al. 2012), remains to be answered. Also, processes to generate such strong large-scale magnetic fields in fully convective objects and to maintain them in largely neutral atmospheres are to be identified.

Among such bursty dwarfs, LSR J1835+3259 (hereafter LSR J1835) is one of the brightest in both near-infrared and quiescent radio flux. Its spectral type M8.5 (Deshpande et al. 2012) places it near the stellar-mass threshold and makes it a possible candidate for one of the warmest brown dwarfs. It 
demonstrates prominent periodic radio bursts, corresponding to a very rapid rotation with the period of only $2.84 \mathrm{hr}$ (Hallinan et al. 2015). Such a fast rotation (three times faster than Jupiter) may indicate the dwarf's young age and, therefore, the lower mass for the given spectral class. No X-ray emission associated with the presence of a magnetically heated corona has been detected so far. We have selected LSR J1835 to test the hypothesis that such bursty brown dwarfs possess strong magnetospheres.

The most reliable way to detect magnetic field on a cool dwarf is to measure its light polarization due to the Zeeman effect, which affects both atomic and molecular lines in its spectrum. In this paper, we present a quantitative analysis of a set of spectropolairmetric data for LSR J1835 taken at the Keck telescope (Harrington et al. 2015). We report the first detection of a strong magnetic field on this brown dwarf. Our results provide direct evidence that strong transient optical and radio emission bursts on such objects are powered by the surface magnetic field of several $\mathrm{kG}$, which is associated with an active region producing both optical and radio emission.

The paper is structured as follows. In Section 2, we briefly describe observations and their reduction and calibration procedures. In Section 3, we analyze spectral feature evolution and evaluate the significance of polarimetric signatures in the $\mathrm{Na}$ I $819 \mathrm{~nm}$ lines, which provide evidence for the surface magnetic field detection. In Section 4, we determine atmospheric parameters of LSR J1835 from the NaI $819 \mathrm{~nm}$ Stokes $I$ line profiles. This helps us to deduce the dwarf's mass and age from evolutionary tracks and establish that LSR J1835 is a young brown dwarf. In Section 5, we present emission region maps obtained by inversions of optical emission line profiles. The spatial distribution of the emission indicates the presence of emission loops rooted in the surface magnetic region and extended above the surface. In Section 6, we model the Na I $819 \mathrm{~nm}$ Stokes $I$ and $V$ line profiles taking into account the Paschen-Back effect and deduce a $5 \mathrm{kG}$ surface magnetic field on LSR J1835. In Section 7, we discuss the significance of our results in the context of magnetic activity in low-mass stars and possible magnetic interactions of the dwarf with its environment (disk, planets). Finally, in Section 8, we summarize our results and conclusions.

\section{Observations}

We carried out spectropolarimetric measurements during $6 \mathrm{hr}$ on two consecutive nights ( $3 \mathrm{hr} /$ night) on 2012 August 22 and 23 with the Low Resolution Imaging Spectropolarimeter (LRISp) at the $10 \mathrm{~m}$ Keck telescope, Mauna Kea, Hawaii. Measurements were made first at four angles of the half-wave plate to obtain linear polarization described by Stokes $Q / I$ and $U / I$ and then at two angles of the quarter-wave plate to obtain circular polarization described by Stokes $V / I$. The individual exposure time was 10 minutes for each position of the wave plates. Thus, we obtained 36 Stokes $I$ flux spectra and six measurements for each of the polarized Stokes $Q / I, U / I$, and $V / I$ during two complete rotation periods of LSR J1835 separated by about $21 \mathrm{hr}$, or about seven rotational periods. The measurements were made simultaneously in the blue $(380-776 \mathrm{~nm})$ and red (789-1026 nm) arms of LRISp, with the spectral resolution of $0.6 \mathrm{~nm}$ and $0.3 \mathrm{~nm}$, respectively.

The data and their reduction and calibration procedures were presented and discussed in detail by Harrington et al. (2015). The reduction procedure included flat-fielding, spectral order extraction, wavelength calibration using standard spectrum lamps, removing cosmic-ray hits, removing spectral ripples due to optical interference using standard star spectra and Fourier analysis, polarimteric calibration, and Stokes parameter calculation. Since the LRISp is a dual-beam polarimeter, combining two-beam Stokes measurements taken at different angles of the wave plates significantly reduces systematic errors. Our thorough reduction procedure has allowed us to achieve the noise standard deviation for both linear and circular polarization of about $1 \times 10^{-3}(0.1 \%)$ per pixel.

Our data set includes observations of LSR J1835 and several standard stars (Harrington et al. 2015), including the active M3.5 dwarf EV Lac. The EV Lac polarized spectra are analyzed here to demonstrate that our reduction procedure is adequate to detect a few $\mathrm{kG}$ field such as that observed on EV Lac (Johns-Krull \& Valenti 1996).

\section{Data Analysis}

Because of the target's rapid rotation and low brightness, circular and linear polarization could not be measured (quasi-) simultaneously. Therefore, they were analyzed separately. Here we present an analysis of observed flux variability of several optical emission lines ( $\mathrm{H} \alpha, \mathrm{H} \beta, \mathrm{H} \gamma$, and $\mathrm{Na} \mathrm{I} \mathrm{D}_{1}$ and $\mathrm{D}_{2}$ lines) in the blue-arm spectra and polarization in the near-infrared (NIR) neutral sodium lines $\mathrm{Na}$ I at $819-820 \mathrm{~nm}$ in the red-arm spectra. Several bands of the $\mathrm{TiO}, \mathrm{CrH}$, and $\mathrm{FeH}$ molecules were also observed in the NIR at $850-1000 \mathrm{~nm}$. Modeling their polarization using previously developed techniques (Berdyugina et al. 2003, 2005; Afram et al. 2007, 2008; Kuzmychov \& Berdyugina 2013) provides additional important constraints on magnetic region properties, which is the subject of a separate paper (Kuzmychov et al. 2017).

On the first night, 2012 August 22, the optical emission observed in the blue arm have indicated the presence of a "hot spot" (an emission region) centered at the same rotational phase $(\sim 0.0)$ as observed one month earlier by Hallinan et al. (2015) in both optical and radio. We used their ephemeris to calculate rotational phases for our observations. On the following night, 2012 August 23, the emission near the phase 0.0 significantly declined, while near the phase 0.6 (opposite hemisphere) a rise of the emission was seen during the last two exposures. To analyze the emission variability in the blue-arm spectra, we have subtracted the most quiescent state spectrum, observed at the phase of 1.300 during the second night, from the 36 Stokes $I$ flux spectra. The resulting emission profiles are shown in Figure 1. They have been used to recover emission maps as described in Section 5.

The maxima of the blue emission near the phase 0.0 during the first night were accompanied by clear Zeeman effect signatures in circular polarization of the NIR Na I lines at 819-820 nm (Figure 2), which appears as a doublet. These are the strongest (and the only relatively unblended) atomic features in the red part of the observed polarized spectrum. In particular, significant Stokes $V / I$ profiles were detected in both lines during the active state at the two phases $0.11-0.19$ (in the beginning of the night) and 1.02-1.10 (3 hr later) on August 22. The lower activity state Stokes $V / I$ averaged over the remaining four circular polarization measurements showed no signal above the $1 \sigma$ noise level. The Stokes $Q / I$ and $U / I$ near the active state may have had some signals too, but their significance in the individual exposures is low. When averaged over the six corresponding measurements, no significant 

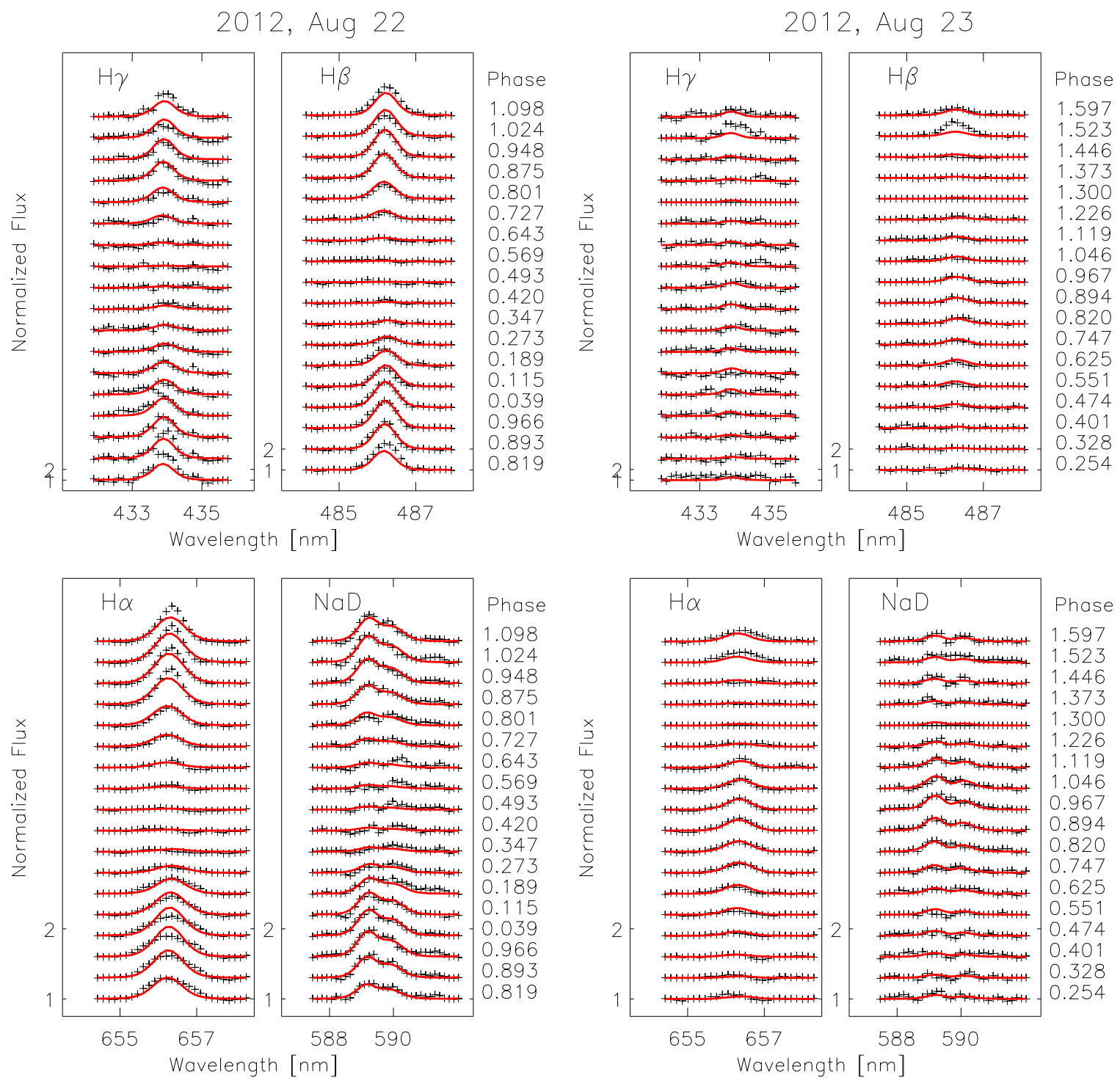

Figure 1. Non-thermal optical emission profiles observed on LSR J1835 during two nights 2012 August 22 and 23 (on the left and right, respectively). The line profiles are obtained by subtracting the quietest state spectrum observed at the rotational phase 1.300 on 2012 August 23, to remove photospheric lines. Crosses are observations, solid lines are best-fit profiles obtained from inversions as described in Section 5. The maximum emission was observed on 2012 August 22, near the phase 0.0. At nearly the same time, the magnetic field signal in the NIR Na I lines Stokes V/I profiles was detected (phases 0.115-0.189 and 1.024-1.098, on 2012 August 22, see Figure 2). On the second night, the emission of the primary active region (near phase 0.0 ) has reduced, and a new emission region has emerged at the opposite side of the dwarf (near phase 0.6).

polarization was found in these profiles. This could also be in part because of cancellation of the opposite polarity signals in different rotational phases. The average Stokes V/I profiles are shown in Figure 2.

Since the spectral PSF is oversampled (Harrington et al. 2015), binning the Stokes $V / I$ spectrum by 3 pixels reduces the noise per spectral bin down to $6 \times 10^{-4}(0.06 \%)$ without a loss of the spectral resolution. The Stokes $V / I$ peak-to-valley average amplitude detected during the active state of LSR $\mathrm{J} 1835$ is $6.2 \times 10^{-3}(0.62 \%)$, see Figure 2 , so the detection significance is about five standard deviations for one Stokes $V / I$ lobe. Considering that we measure two profiles with four lobes, the overall significance of this detection is higher.

On the second night, the optical emission near phase 0.0 has significantly decreased, and no magnetic signal in the NIR Na I lines was observed (within the noise level), thus, indicating a possible decay of the magnetic region. During the last two exposures, the emission started to increase at the opposite hemisphere (near phase 0.5), indicating a possible emergence of a new bursting region. Yet the low hydrogen emission level was not accompanied by a detectable magnetic signal in the NIR Na I lines.

It is worth mentioning that the NIR Na I and optical $\mathrm{Na}_{\text {I }} \mathrm{D}_{1}$ and $\mathrm{D}_{2}$ share the same electronic doublet $P$-state (with the orbital momentum $L=1$, spin $S=1 / 2$, and principal quantum number $n=3$ ). This is the upper state for the optical Na I lines and the lower state for the NIR Na I lines. Therefore, we have also searched for emission and absorption variations in the NIR line. There were no variations found above the noise level. Thus, we conclude that the detected Stokes $V / I$ profiles are formed due to absorption in the magnetized photosphere. This is supported by the fact that the polarization sign observed in the NIR Na I lines and in absorption $\mathrm{CrH}$ lines (Kuzmychov et al. 2017) is the same.

To verify our calibration procedure, we also measured polarization of a very active, flaring M3.5 red dwarf EV Lac 


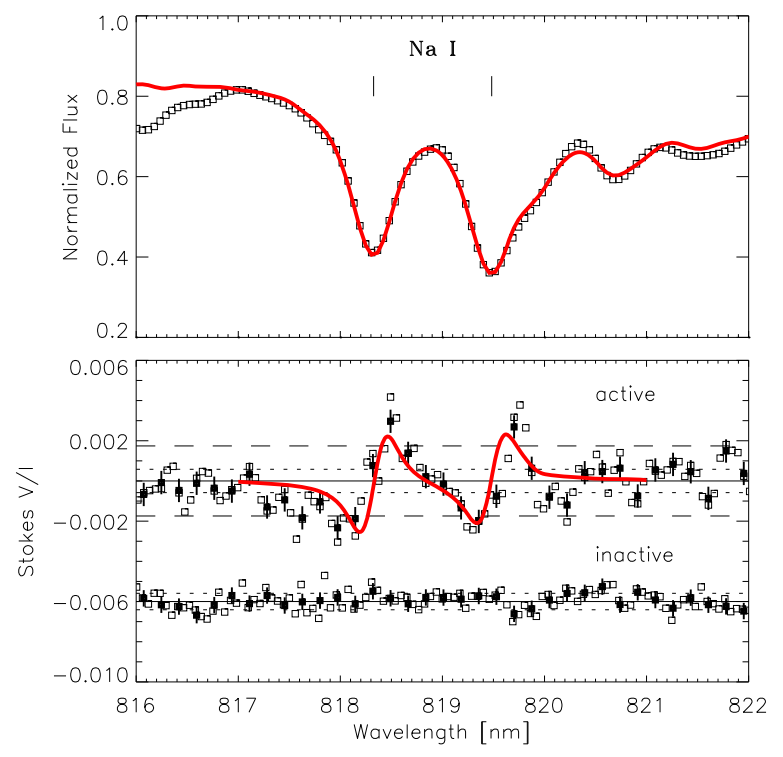

Figure 2. NIR Na I line profiles of LSR J1835: observed (symbols) and modeled (thick red lines). Thin symbols are unbinned data, and thick symbols with error bars are binned by a factor of three. The model profiles are shown for $T_{\text {eff }}=2800 \mathrm{~K}, B=5.1 \mathrm{kG}$, and $f=0.11$. The observed normalized flux spectrum (top panel) is averaged over all measured Stokes $I$ spectra (SNR 6000). In the bottom panel, average Stokes $V / I$ profiles are shown. The top Stokes $V / I$ spectrum is an average of two measurements near the phase 0.0 obtained on 2012 August 22, corresponding to the active state of the dwarf (see Figure 1). The bottom observed Stokes $V / I$ spectrum is an average of four measurements at other phases, corresponding to an inactive state of the dwarf. Horizontal solid lines indicate zero polarization, while dotted and dashed lines are the $\pm 1 \sigma$ and $\pm 3 \sigma$, respectively, noise levels per pixel.

during our observing run. This star is known to possess a magnetic field of $3.8 \pm 0.5 \mathrm{kG}$ with at least $50 \pm 13 \%$ coverage of the stellar surface (Johns-Krull \& Valenti 1996). We detected Stokes $V / I$ peak-to-valley amplitude of $9.6 \times 10^{-3}(0.96 \%)$. No significant Stokes $Q / I$ or $U / I$ were detected. The noise per pixel achieved is $9 \times 10^{-4}(0.09 \%)$. The Stokes profiles are shown in Figure 3). Our measurement for EV Lac agrees with the result by Johns-Krull \& Valenti (1996; see Section 6), assuming that field is predominantly radial near the center of the visible stellar disk.

\section{LSR J1835+3259 Atmosphere Parameters, Mass, and Age}

To determine the atmosphere parameters of LSR J1835, we compute Stokes profiles with the STOPRO code capable of solving a full set of polarized radiative transfer equations for both atomic and molecular lines including magnetic field effects and under the LTE assumption (Frutiger et al. 2000; Berdyugina et al. 2003). To evaluate possible systematic errors, we have used three grids of model atmospheres with the solar metallicity and other element abundances: the PHOENIX models AMES-Cond (Allard et al. 2001) with clear atmospheres and BT-Settl with clouds (Allard et al. 2012) as well as MARCS clear models (Gustafsson et al. 2008).

The NIR Na I lines are blended by numerous weak molecular lines. We have identified major contributions from the TiO bands of the triplet $\gamma$ and singlet $\delta$ systems. In particular, the strongest band heads were observed at $820 \mathrm{~nm}$ from the $\delta(1,0)$ band and at $820.5 \mathrm{~nm}$ from the $\gamma(2,1)$ band. Overall, we have included about 7000 lines from the laboratory list of Davis et al. (1986) and simulated list by Plez (1998). Magnetic level

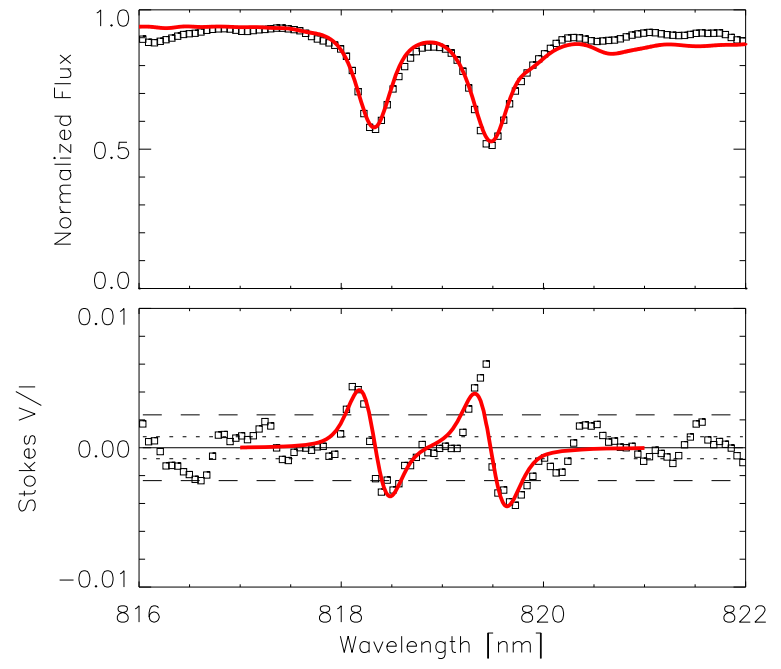

Figure 3. Same as Figure 2 for EV Lac. The model Stokes profiles are shown for $T_{\text {eff }}=3300 \mathrm{~K}, B=3.8 \mathrm{kG}, \gamma=0^{\circ}$, and $f=0.28$.

splitting and transition probabilities were calculated using a perturbation theory described by Berdyugina \& Solanki (2002) and Berdyugina et al. (2005). This theory is accurate to explain Stokes profiles in the TiO lines observed with high resolution in sunspots (Berdyugina et al. 2000, 2006a) and starspots (Berdyugina et al. 2006b). We have also verified wavelengths of simulated lines by comparing the list with high-resolution spectra of $\mathrm{M}$ dwarfs obtained with the ESPADONS spectropolarimeter at the CFHT (Berdyugina et al. 2006b). At the spectral resolution of our data, we do not detect significant $\mathrm{TiO}$ polarization due to blending. However, their absorption influences Stokes profile shapes of the $\mathrm{Na}$ I lines.

By simultaneously modeling the $\mathrm{Na} \mathrm{I}$ and $\mathrm{TiO}$ lines in the $816-822 \mathrm{~nm}$ regions, we have found that this region is extremely sensitive to both the effective temperature $T_{\text {eff }}$ and gravitational acceleration $\log g$. In particular, the $\mathrm{Na}$ I line wings are mostly sensitive to the gravity, while the weak $\mathrm{TiO}$ bands are sensitive to the temperature (Figure 4, upper panel). These features form in deep atmospheric layers, so they reliably probe the gravity and temperature near optical depth 1, i.e., in the photosphere. Earlier, the sensitivity of these lines to the gravity and age was pointed out by Luhman (2012) based on a comparison of line profiles for late-M young cluster members and field dwarfs. We have searched for the best fit to the observed Stokes $I$ normalized flux profiles within each of the three model grids by means of minimization of the $\chi^{2}$ functional, according to Press et al. (2007).

All the model grids have shown well-constrained $\chi^{2}$ minima for the model with $T_{\text {eff }}=2800 \mathrm{~K}$ and $\log g=4.5$ (Figure 4, lower panel). The confidence levels for the three model grids nicely overlap around the minimum. For the clear atmospheres, such as the AMES-Cond and MARCS-SG models, contours are almost identical. This allows us to narrow down the uncertainties to $\pm 30 \mathrm{~K}$ for $T_{\text {eff }}$ and \pm 0.05 for $\log g$. We note that non-LTE effects were not accounted for in our modeling, but they may not influence our result, because we have achieved a good fit to both weak molecular lines and strong $\mathrm{NaI}$ lines. For EV Lac, we found the best fit for the model with $T_{\text {eff }}=3300 \mathrm{~K}$ and $\log g=4.5$, i.e., what can be expected for an M3.5 dwarf with cool spots.

We note that strong molecular bands, such as $\mathrm{TiO}, \mathrm{CrH}$, and $\mathrm{FeH}$ bands in the 830-1000 $\mathrm{nm}$ region (included in our data), are 

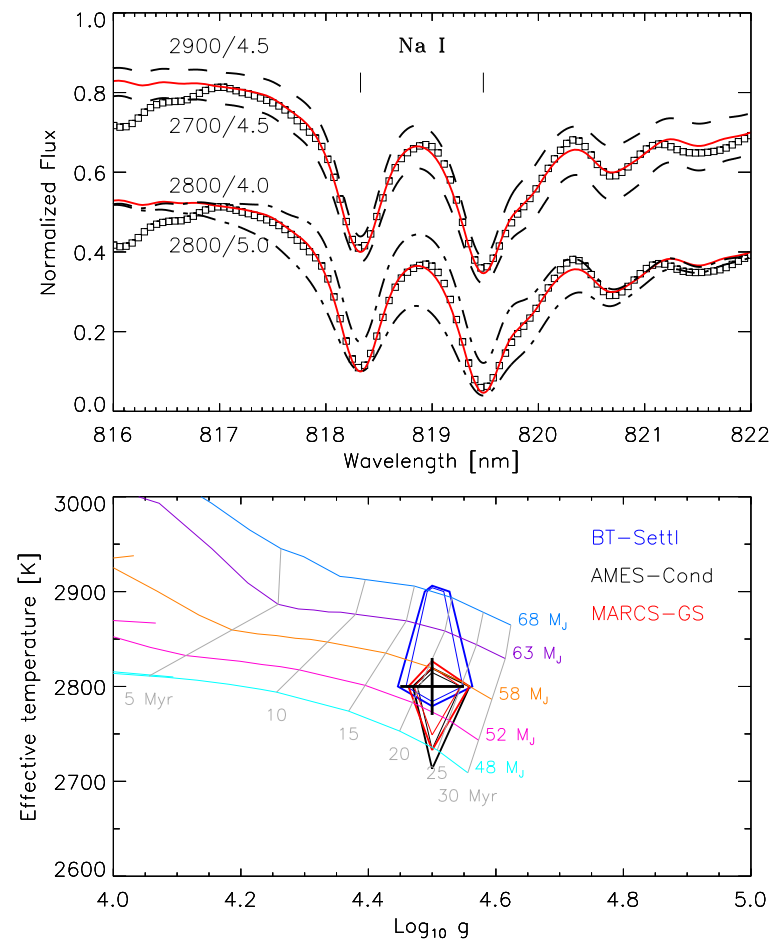

Figure 4. Top panel: the best-fit model spectra for $T_{\mathrm{eff}}=2800 \mathrm{~K}$ and $\log g=4.5$ (solid red lines) compared to the observed $\mathrm{Na} \mathrm{I}+$ TiO Stokes $I$ normalized flux line profiles (squares) and the model spectra for $T_{\mathrm{eff}}=2700 \mathrm{~K}$ and $2900 \mathrm{~K}$ (dashed lines) and $\log g=4.0 \log g=5.0$ (dashed-dotted lines) using AMES-Cond models. Lower spectra are shifted down by 0.3 for clarity. Bottom panel: the confidence contours for the three model grids AMES-Cond (black), BT-Settl (blue), and MARCS-SG (red) in the $\chi^{2}$-landscape. The $67 \%$ level $(1 \sigma)$ is the thin inner contour, and the $99 \%$ level $(3 \sigma)$ is the thick outer contour. The minima for the three model types overlap at the best-fit solution and define its uncertainty (marked with the cross). Contours for the AMES-Cond and MARCS-SG models are almost identical. In addition, evolutionary tracks by Baraffe et al. (2017) for young brown dwarfs with different masses (thin solid curves of various colors) and corresponding isochrones (light gray solid lines) are shown and labeled. The atmospheric parameters of LSR J1835 correspond to a brown dwarf with the a mass of $55 \pm 4 M_{\mathrm{J}}$ and an age of $22 \pm 4 \mathrm{Myr}$.

also sensitive atmospheric diagnostics. However, they contain a significant contribution from cool magnetic starspots that increases the number of unknowns and their errors. In addition to the temperature sensitivity, metal hydrides are known to be quite sensitive to the gravity acceleration (e.g., Bell et al. 1985; Schiavon et al. 1997), and they are also highly magnetically sensitive (Berdyugina et al. 2005; Afram et al. 2008; Kuzmychov \& Berdyugina 2013). Our analysis of the observed Stokes $I$ in these molecular bands results in the temperature of the non-magnetic photosphere $T_{\text {phot }}=2900+150 /-250 \mathrm{~K}$, magnetic spot temperature $T_{\text {spot }}=2200+250 /-150 \mathrm{~K}$, spot filling factor $f_{\text {spot }}=0.6$, and $\log g$ of 4.5-5.0, equally good within the errors (Kuzmychov et al. 2017). The $T_{\text {eff }}=2800 \mathrm{~K}$ and $\log g=4.5$ determined in the present paper are more accurate, because they are based on weak, higher-excitation $\mathrm{TiO}$ bands and wings of the subordinate atomic lines (also higherexcitation lines), which form deeply in the atmosphere and are less sensitive to starspots.

Knowing the atmosphere parameters, we can now deduce the age and mass of LSR J1835 from evolutionary models. Different models, e.g., by Burrows et al. (1997) and Baraffe et al. (2017), indicate a young age of the dwarf. The models by
Baraffe et al. (2017) are based on self-consistent calculations, which couple numerical hydrodynamics simulations of collapsing pre-stellar cores and stellar evolution models of accreting low-mass stars and brown dwarfs with the age up to $30 \mathrm{Myr}$. Models with two accretion scenarios were considered: a cold accretion model, with all accretion energy is radiated away, and a hybrid accretion, with the amount of accreted energy depending on the accretion rate. In particular, the hybrid scenario helps explain observed luminosity spread in young clusters and FU Ori eruptions. Using these models, we determine that an object with $T_{\text {eff }}=2800 \pm 30 \mathrm{~K}$ and $\log g=4.50 \pm 0.05$ is a young brown dwarf with the mass, radius, and age as follows:

$$
M=55 \pm 4 M_{\mathrm{J}}, R=2.1 \pm 0.1 R_{\mathrm{J}}, t=22 \pm 4 \mathrm{Myr} .
$$

Relevant evolutionary tracks and isochrones for the hybrid accretion scenario are shown in the lower panel of Figure 4, but the differences between the cold and hybrid scenario tracks are significantly smaller than our error bars. For comparison, the older models by Burrows et al. (1997) predict a similar age (27 Myr) but a somewhat smaller mass $\left(44 M_{\mathrm{J}}\right)$ and radius $\left(1.9 R_{\mathrm{J}}\right)$. Therefore, we conclude that LSR J1835 is a young brown dwarf in the end of accretion phase (see also Section 7).

We emphasize that LSR J1835 atmosphere parameters significantly differ from those typical for late stellar-mass $\mathrm{M}$ dwarfs. For instance, a red dwarf star with $T_{\text {eff }}=2800 \mathrm{~K}$ would have the spectral class M5 and $\log g=5.0$, while an M8 star with the mass $0.08 M_{\odot}$ would have $T_{\text {eff }}=2400 \mathrm{~K}$ and $\log g=5.25$. As concerns the kinematics, UVW velocities of brown dwarfs in the solar neighborhood are rather disperse (Zapatero Osorio et al. 2007), and LSR J1835 falls in the middle of the distribution.

\section{Emission Maps and Loops}

The two time series of the emission line profiles obtained during two complete rotations of the dwarf (Figure 1) are suitable for recovering the topology of the emitting regions on LSR J1835 using an indirect Doppler imaging technique. Here we employ the inversion method based on Occam's razor principle by Berdyugina (1998), which was successfully employed for indirect imaging of cool active stars using spectroscopic and photometric measurements (e.g., Berdyugina 2005).

Inversion techniques, when applied to spectra, rely on the knowledge of local line profiles depending on physical conditions (e.g., temperature, density, magnetic field, etc.) at a given location on the surface. Such profiles have recently been computed using the most advanced NLTE radiativehydrodynamic model of a typical M-dwarf flare by Kowalski et al. (2015). They have demonstrated that the F13 flare model based on precipitating non-thermal electrons with the energy flux of $10^{13} \mathrm{erg} \mathrm{cm}^{-2} \mathrm{~s}^{-1}$ successfully reproduces the color temperature and Balmer jump ratio observed in M-dwarf highly energetic flares.

We have found that this model is also suitable for interpreting emission profiles of LSR J1835. In particular, we consider model line profiles at rising, peak, and decaying stages of the flare (RF, $\mathrm{PF}$, and DF, respectively). These profiles differ by equivalent widths and broadening in the line cores (Doppler broadening) and in the line wings (Lorentz broadening) due to evolution of the plasma temperature and density during the flare. 

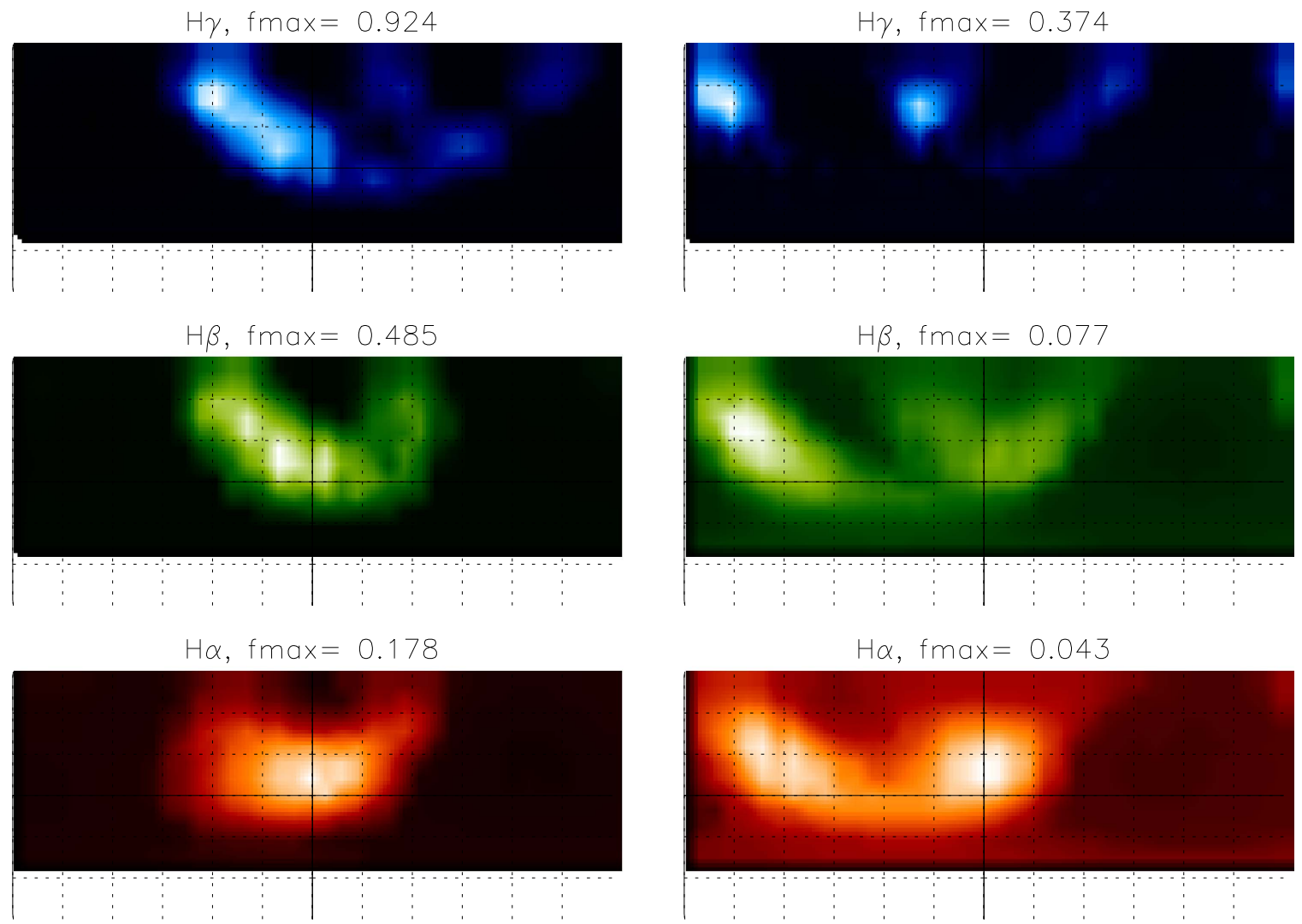

$\mathrm{NaD}, \mathrm{fmax}=0.162$

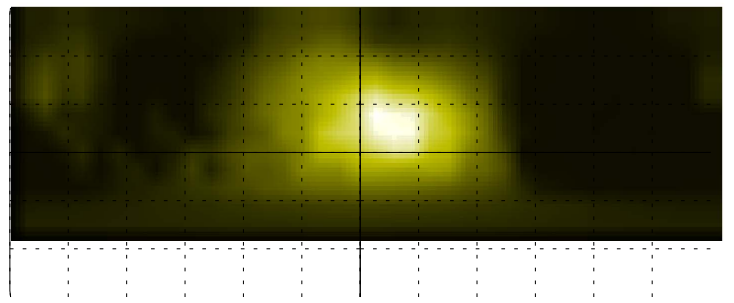

$\mathrm{NaD}, \mathrm{fmax}=0.053$

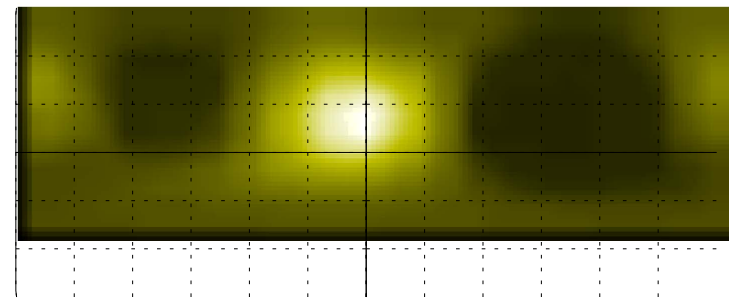

Figure 5. Non-thermal optical emission maps recovered from the profiles shown in Figure 1 during two nights 2012 August 22 and 23 (on the left and right, respectively). Maps are plotted in a cylindrical projection: the coordinate grid is shown with the $30^{\circ}$ interval in both latitude and longitude (dotted lines). The phase $=0.0$ meridian and the equator are shown with solid lines. The inclination of the rotational axis is $50^{\circ}$. The maximum filling factor of the hot flare-like plasma (see Section 5) is provided above each image and corresponds to the lightest area in the images. The minimum filling factor (0.0) corresponds to the darkest areas in the images.

Here we summarize most relevant characteristics of the flare stages and refer to other details in Kowalski et al. (2015). The model predicts that most of the non-thermal electron energy is first deposited into hydrogen ionization and then into raising the temperature, up to $180,000 \mathrm{~K}$, resulting in massive ionization of hydrogen and helium (and other elements) and a sequence of explosive events during the rising stage of the flare (models RF, F13 0.2s and 1.2s). At the peak of the flare (model PF, F13 2.2s) the temperature settles at $12,500 \mathrm{~K}$ and $13,000 \mathrm{~K}$, and then eventually drops to $7000 \mathrm{~K}$ at the decaying stage of the flare (model DF, F13 4s). Correspondingly, the continuum and line emission are observed due to recombinations and radiative decays during the flare. As the flare decays, the emission decreases and the line profiles become narrower. Note that the timescale of the model flare $(0.2 \mathrm{~s}$ to $4 \mathrm{~s}$ after the flare peak) is too short as compared to flare durations in $\mathrm{M}$ dwarfs and the brown dwarf discussed here. This can be overcome by integrating over a series of flaring events triggered by an initial reconnection (Kowalski et al. 2015).
Nevertheless, this model provides us with realistic emission line profiles at different flare stages suitable for our analysis.

We have carried out inversions of the time series line profiles for each night and each emission line separately, thus obtaining four maps corresponding to different heights in the dwarf atmosphere (according to the response of the atmosphere to the flare) for each full rotation. They are presented in Figure 5. A relatively low-resolution map coordinate grid of $6^{\circ} \times 6^{\circ}$ was used to reduce the number of independent parameters. The emission flux from each map pixel was calculated using one selected model line profile for a given emission line weighted by a filling factor $f$. Thus, the recovered image is a map of the filling factor for a given model emission line. This approach allows us to identify the activity stage of the emission bursts observed on LSR J1835 and evaluate the temperature and density needed to produce such emission.

Out of all the F13 model line profiles, the decaying flare stage profiles (DF, F13 4s) were found on average to provide the best fits to the observed profiles. The PF and RF profiles 

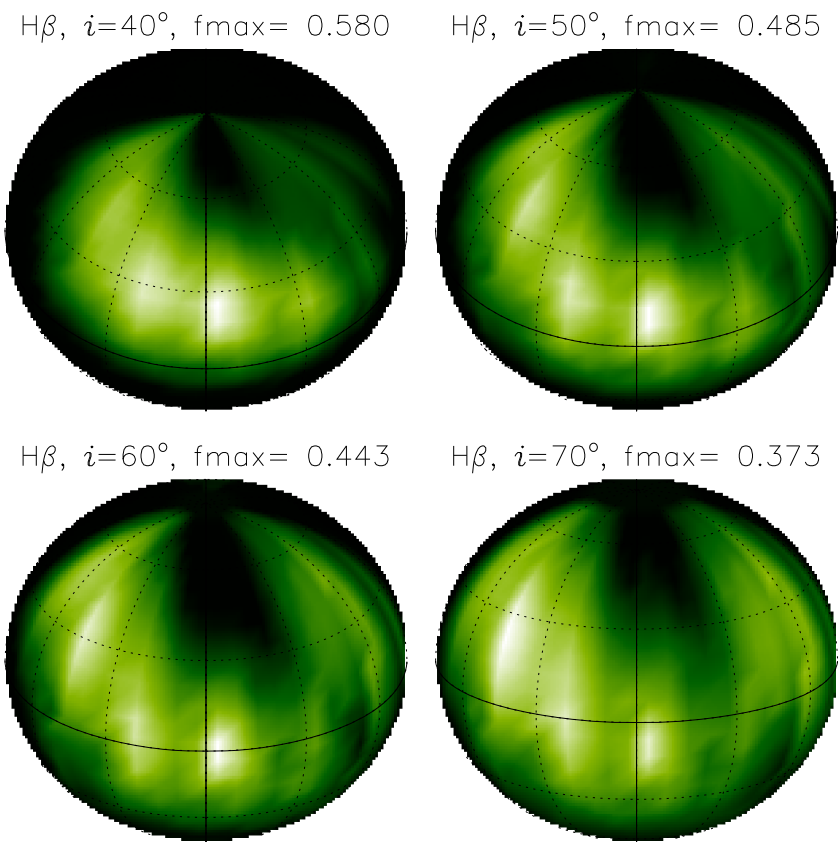

Figure 6. $\mathrm{H} \beta$ emission maps as in Figure 5 for different inclination angles of the dwarf's rotational axis, for the night 2012 August 22. The images are shown at the phase 0.0 in an orthographic projection, i.e., this is how an observer would see the dwarf's surface for a given inclination angle. The dwarf is assumed to rotate clock-wise as seen from the visible pole.

were found to be too broad (plasma is too hot and too dense) to obtain satisfying fits to the observed profiles. Finer details in the emission evolution were found to deviate also from the best DF F13 4s model: in the beginning of observations the measured profiles are somewhat broader than the model (indicating temperature $>7000 \mathrm{~K}$ and higher density), while toward the end of observations the measured profiles are narrower (indicating temperature $<7000 \mathrm{~K}$ and lower density). We found that by adjusting Lorentz broadening we can achieve better fits. This is simply because a brown dwarf atmosphere is more compact than an early M-dwarf atmosphere, leading to a smaller scale height and larger density gradient. Furthermore, temperature and density are also expected to evolve as the emission slowly decays. Most recently (after our analysis was completed) the F13 model was further updated by Kowalski et al. (2017) with a new electric pressure broadening prescription allowing for broader emission lines. This theoretical improvement is in agreement with our empirical adjustments.

The dwarf's projected rotational velocity $v \sin i$ of $50 \pm 5 \mathrm{~km} \mathrm{~s}^{-1}$ has been estimated from fits to the NIR Na I flux profiles (Section 6). With this rotational velocity and the spectral resolution of $0.5-0.6 \mathrm{~nm}$ in the blue arm, there is no measurable Doppler shifts to resolve emission latitudes. However, since the dwarf rotation is much faster than the emission decay, the emission amplitude rotational modulation and its visibility during a range of phases provide a good constraint on its longitude and somewhat limited constraint on the latitude (in fact, they are projected latitude and longitude, since emission is formed above the photosphere). The longitudinal resolution is about $10^{\circ}$ due to the 10 minute sampling of the profiles.

The inclination of the rotational axis to the line of sight is unknown. To investigate the sensitivity of the result to this parameter, we obtained maps for the inclinations of $40^{\circ}, 50^{\circ}, 60^{\circ}$, and $70^{\circ}$. Maps for the $\mathrm{H} \beta$ emission at different inclination angles are presented in Figure 6 as examples. The variations of the active region geometry depending on the inclination are typical for Doppler imaging (see Berdyugina 1998), i.e., the emission latitudes and maximum filling factors adjust themselves accordingly to maintain the same emission flux at a given rotational phase. Hence, the emission region area increases with the inclination angle, while the maximum filling factor decreases. Since the emission is observed at a wide range of rotational phases, at lower inclination its area is compact and shifted to higher latitudes, while at higher inclinations the emission region is broad and shifted to lower latitudes. However, the relative distribution of the emission in different spectral lines remains about the same, which is an important constraint on the topology of the emitting region.

The recovered emission maps indicate the presence of dense and hot plasma, of at least $7000 \mathrm{~K}$, which is much hotter than the surrounding atmosphere. The relative latitude of the region near the phase 0.0 varies depending on the line: emission of the higher-excitation lines $(\mathrm{H} \gamma$ and $\mathrm{H} \beta)$ occurs at higher latitudes and occupy a larger area, while emission of the low-excitation lines $\left(\mathrm{NaI} \mathrm{D}_{1}\right.$ and $\mathrm{D}_{2}$ ) occupies a compact region at lower latitudes. The emission in $\mathrm{H} \alpha$ is intermediate to these two extremes with the brightest spot close to that of the $\mathrm{NaI} \mathrm{D}$ lines.

We visualize the spatial connection of the emission in different lines by overplotting contours around their maxima in Figure 7. There are at least two emission loops (marked as L1 and L2) within the primary region near the phase 0.0 on the first observing night. The emission is apparently vertically stratified within the loops. These loops are seen under different angles at different rotational phases and rotate with the dwarf in and out of view causing the emission bursts. In the true, orthographic projections (Figure 6) the loops are reminiscent of large filaments/prominences above the dwarf's surface, as directly observed on the Sun. Such loops may extend one to three dwarf radii from the surface (Lynch et al. 2015). The region near the phases $0.4-0.5$ on the second night seems have a vertical structure similar to that of the primary region.

One month earlier, observations by Hallinan et al. (2015) detected emission activity near the phase 0.0 . This provides a lower limit on the life time of the region. For comparison, on the Sun, large prominences can last several months and are often sources of large eruptions. As described in Section 2, the phase of the emission region on LSR J1835 coincides with the phase at which the Stokes $V / I$ was detected in the NIR Na I lines. Thus, the emission is clearly associated with a magnetic field. We model this magnetic signal in the following section.

\section{Magnetic Field}

\subsection{Paschen-Back Effect in the Na I lines}

The NIR Na I line parameters (wavelength, lower level excitation energy, oscillator strengths, and electronic configurations) were taken from the Kurucz database (Kurucz 1993). They indicate that the upper doublet levels with $J^{\prime}=2.5$ and 1.5 (the fine structure levels) are split only by $0.05 \mathrm{~cm}^{-1}$. This implies that magnetic splitting of these levels will be comparable to the fine structure splitting at the field strength of $200 \mathrm{G}$. Therefore, at stronger fields the magnetic 

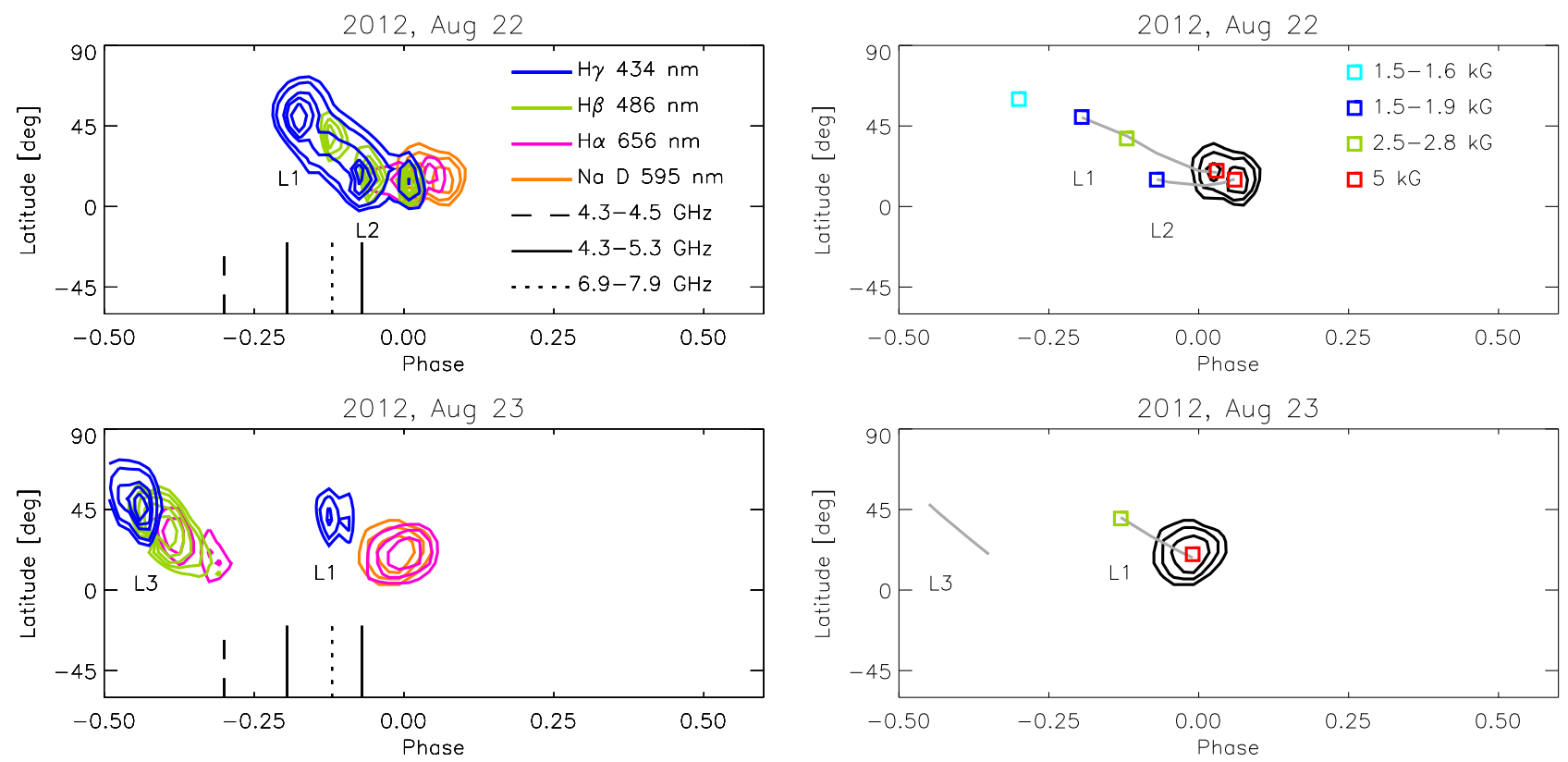

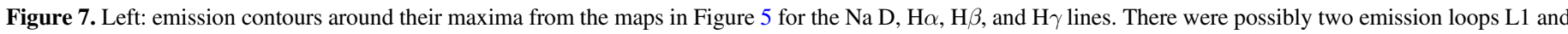

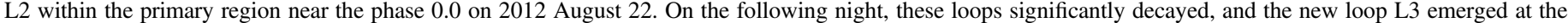

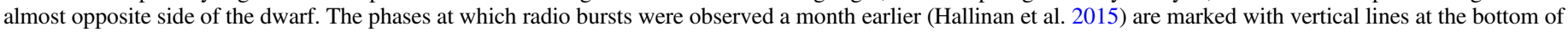

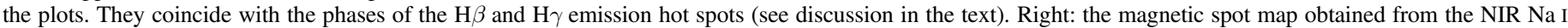

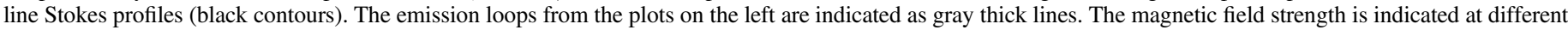
locations along the loops as deduced from the the $\mathrm{Na}$ I Stokes profiles and the radio frequencies of the bursts.

perturbation should be considered in the intermediate PaschenBack regime (PBR). The lower doublet levels with $J^{\prime \prime}=1.5$ and 0.5 are split by about $17 \mathrm{~cm}^{-1}$, which requires fields stronger than $100 \mathrm{kG}$ for the PBR. Therefore, magnetic perturbations of the lower levels can be safely described in the linear Zeeman regime (ZR).

It is easy to solve the intermediate PBR problem for doublet atomic levels, as described in textbooks (e.g., Sobelman 1992). We show a few examples of level splitting of the NIR Na I lines in Figure 8. Several interesting effects in the PBR are worth to noting: (1) mixing of the fine structure levels (i.e., $J^{\prime}$ is no longer a good quantum number), (2) mixing of magnetic components of different lines, (3) change of permitted line strengths as compared to the zero-field values, and (4) appearance of forbidden transitions (here, with $\Delta J=J^{\prime}-J^{\prime \prime}=2$ ). As a result, line splitting undergoes complex nonlinear transformations as the field strength increases. Therefore, taking into account these effects is important for adequate interpretation of observed Stokes profiles.

Example synthetic Stokes $V / I$ profiles of one Na I line are shown in Figure 9 for three magnetic strength values. To emphasize the PBR effect, we also show profiles computed in the ZR, i.e., by neglecting level mixing. As expected, the difference is especially noticeable at stronger fields. It is important to notice that the line shape also varies and cannot be approximated using the weak-field assumption. In fact, shapes of the Na I Stokes $V / I$ profiles and their relative amplitudes provide robust constraints on the field strength. If Stokes $V / I$ in the PBR is measured with a high signal-to-noise ratio (and preferably with a high spectral resolution), it can uniquely identify the field strength, independently on the magnetic field filling factor. We employ this sensitivity of the NIR Na I lines in this paper.

\subsection{Magnetic Field on LSR J1835+3259}

To infer properties of the magnetic field on LSR J1835, we first fit the observed average Stokes $V / I$ profile shown in Figure 2 with the synthetic PBR profiles by varying the magnetic field strength, $B$, and its filling factor, $f$ (i.e., a fraction of the visible surface with the magnetic field) under two assumptions: (1) the field is homogeneous and longitudinal, i.e., along the line of sight, and (2) the field is homogeneous and inclined to the line of sight with the angle $\gamma$. These assumptions make no attempt to constrain the global field or satisfy $\operatorname{div} B=0$. Also, the field direction within the active regions may be neither longitudinal nor homogeneous, if the emission loops in Figure 7 follow magnetic field lines. Nevertheless, the first case provides the lowest limit for the magnetic field strength, and the second one can indicate more realistic estimates. The modeled PBR Stokes $V / I$ for a given field strength and the angle $\gamma$ is simply scaled with the factor $f$ : $V / I(B, \gamma, f)=f \times V / I(B, \gamma, f=1)$. In the cases $(1) \gamma=0^{\circ}$ or $180^{\circ}$. The magnetic region was assumed to be of the same temperature as the non-magnetic atmosphere. A change of the spot temperature would affect the filling factor but we do not expect large variations for cool dwarfs (e.g., Berdyugina 2005).

Since the Na I Stokes profiles are in the PBR at $B>200 \mathrm{G}$, they have sensitivity to disentangle the magnetic field strength and the filling factor for $\mathrm{kG}$ fields, even though this is limited by the SNR of the data. Also, in a strong-field regime, the magneto-optical effect introduces a polarization reversal in the middle of the Stokes $V$ for an inclined field. This allows us to also infer the angle $\gamma$. Examples of such profiles are shown in Figure 9. Hence, we obtain a range of feasible solutions for the three parameters: $B, \gamma$, and $f$.

We have found that the field inclination angle $\gamma$ is well constrained by the Stokes $V$ profile shapes. For $B \geqslant 1 \mathrm{kG}$ (i.e., when the magneto-optical effect is strong), we obtain 

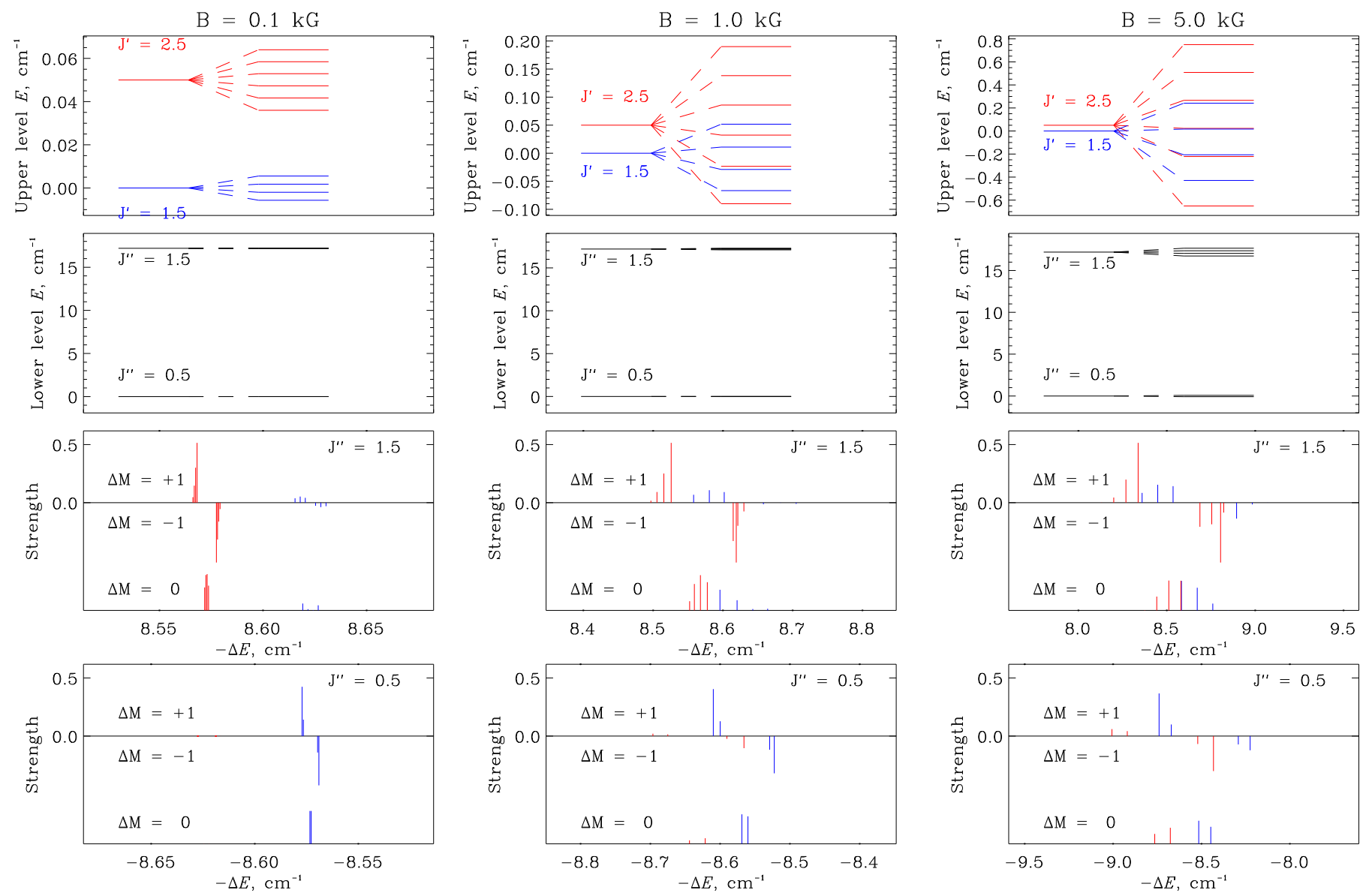

Figure 8. Magnetic splitting of the NIR Na I fine structure levels (top two rows) and magnetic transitions (bottom two rows). The levels and corresponding transitions involving magnetic sublevels of the $J^{\prime}=2.5$ level are shown in red, and those of $J^{\prime}=1.5$ are shown in blue. The magnetic transitions for $\Delta M=+1$ are shown upward, for $\Delta M=-1$ downward, and for $\Delta M=0$ at the bottom of the plots.

$\gamma=130^{\circ} \pm 10^{\circ}$. Correspondingly, for the longitudinal field model, we find $\gamma=180^{\circ}$. Hence, the magnetic field is directed from the observer and most probably inclined with the angle of $130^{\circ}$. This agrees very well with the projected direction of the loops as illustrated in Figure 7. The azimuth of these loops can characterize the azimuth angle $\chi$ of the $B$-field.

In Figure 10, we show envelopes of $(B, f)$ solutions as contours for $\gamma=180^{\circ}$ (solid contour) and $\gamma=130^{\circ}$ (dashed contour). Two immediate conclusions are that the field must be stronger than $1 \mathrm{kG}$ and its filling factor must be $\geqslant 4 \%$.

We can further constrain the field strength in the active region near the phase 0.0 on 2012 August 22 using its area from the Na I D emission contours as shown in Figure 7. For instance, the 0.2 level of the maximum emission filling factor encompasses most of the emission flux. For the phases 0.0 to 0.2 , during which the Stokes $V / I$ profile in Figure 2 was measured and averaged, the area within this level is averaged to $11 \%$ of the visible projected surface area. Now, according to the contours in Figure 10, the longitudinal field in this region is $5.1 \pm 1.3 \mathrm{kG}$. The error on the filling factor from the same contour is \pm 0.04 . An inclined field of the same strength requires $f=0.17 \pm 0.04$ or up to $10 \mathrm{kG}$ for $f=0.11$. We note, however, that the error of $B$ increases for stronger fields. Higher SNR and spectral resolution are needed for obtaining tighter constraints on the field parameters. The Stokes $V / I$ profile corresponding to the $B=5.1 \mathrm{kG}$ and $f=0.11$ is shown with the red line in the lower panel of Figure 2.

We emphasize that this is the first detection of a surface magnetic field on a brown dwarf, which is so far the coolest known dwarf with such strong magnetic fields. Earlier attempts to achieve this were inconclusive. For instance, an extensive study of magnetic properties of a sample of M7-M9 dwarfs by Reiners \& Basri (2010) included only old dwarfs with very modest activity levels. As a result, only a couple of objects were reported to have magnetic fields of $3-4 \mathrm{kG}$ with an approximate error of $\pm 1 \mathrm{kG}$. However, their analysis was based on fitting observed unpolarized flux spectra using a much warmer M3.5 spectrum of EV Lac as the template. This approach leads to unknown biases in the value of the magnetic field strength and its error. In contrast, our approach to observe polarized spectra and carry out detailed polarized radiative transfer taking into account numerous blends provides definite detection and realistic magnetic field parameters.

For EV Lac, we have assumed the field strength of $3.8 \mathrm{kG}$ (Johns-Krull \& Valenti 1996) and obtained the best fit with the filling factor $f=28 \%$ and $\gamma=0^{\circ}$ for a longitudinal field (Figure 3) and $f=43 \%$ and $\gamma=50^{\circ}$ for an inclined field. The second value agrees well with the earlier estimate of the magnetic filling factor of $50 \pm 13 \%$ (Johns-Krull \& Valenti 1996). Parameters for a few very active red dwarfs 


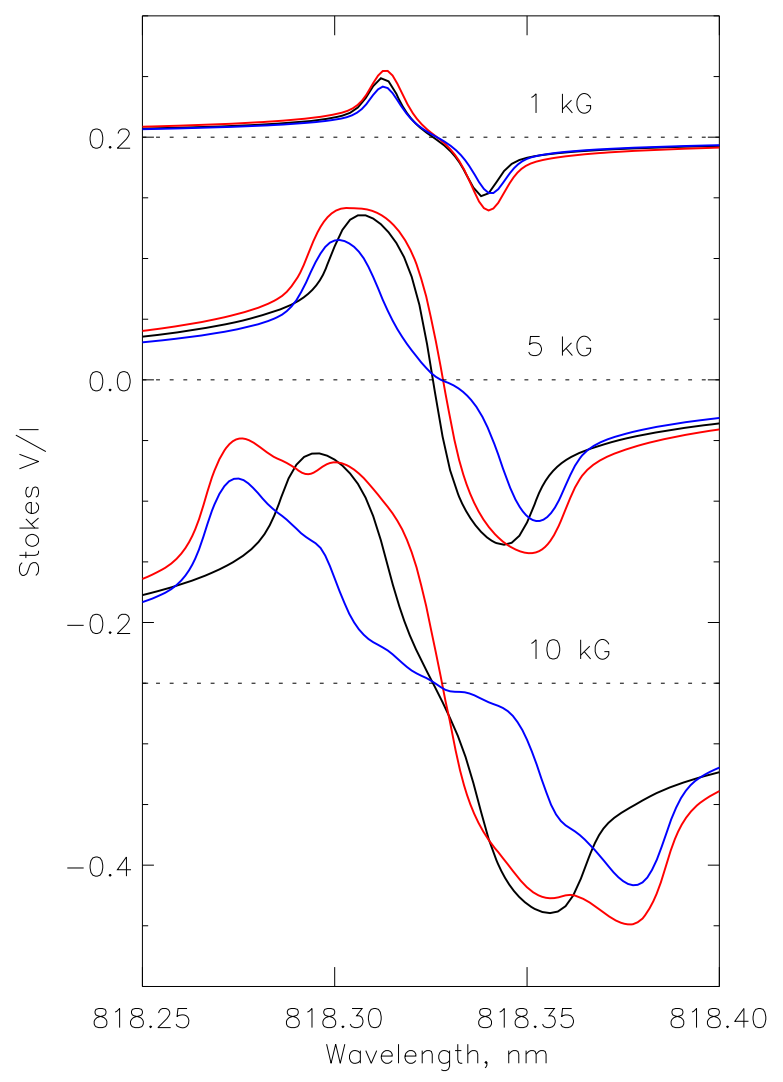

Figure 9. Stokes profiles of the Na I $818.3 \mathrm{~nm}$ line at magnetic field strengths of 1,5 , and $10 \mathrm{kG}$. The profiles computed in the $\mathrm{ZR}$ assuming the field inclination angle $\gamma=0$ are shown with black lines. The profiles computed in the PBR assuming $\gamma=0^{\circ}$ and $40^{\circ}$ are shown with red and blue lines, respectively. The profiles are shifted in vertically for clarity. The corresponding zero levels are shown with dotted lines. The difference between the PBR and ZR profiles and the sensitivity to the $\gamma$ angle due to the magneto-optical effect increase with the field strength.

(including EV Lac) are plotted in Figure 10 for comparison (from Johns-Krull \& Valenti 1996; Berdyugina 2005).

\section{Discussion}

The observed circular polarization signature most probably represents a residual of multiple opposite-sign contributions from a complex field, which may never be spatially resolve (as on red dwarfs), so we can interpret our measurements only in terms of residual homogeneous fields. However, modeling the PBR polarization in the Na I lines of LSR J1835 clearly indicates that the magnetic field in its active region should be stronger than $1 \mathrm{kG}$ (Figure 10). An equipartition estimate of about $5 \mathrm{kG}$ can be inferred from the LSR J1835 model atmosphere assuming that the magnetic field strength should scale with the local gas pressure in the photosphere. In the solar atmosphere this value is about $1.5 \mathrm{kG}$, and such fields are observed as small-scale magnetic elements in hot active regions (plages), while cool sunspots can harbor a factor of two to three stronger fields, i.e., $3-4.5 \mathrm{kG}$. On EV Lac, the equipartition magnetic field is $3.1 \mathrm{kG}$ at the base of its atmosphere, which is a bit lower than the observed $3.8 \pm 0.5 \mathrm{kG}$. Our circular polarization measurements imply that a $5.1 \pm 1.3 \mathrm{kG}$ longitudinal field on LSR J1835 could occupy $11 \pm 4 \%$ of the visible hemisphere and an inclined field would occupy

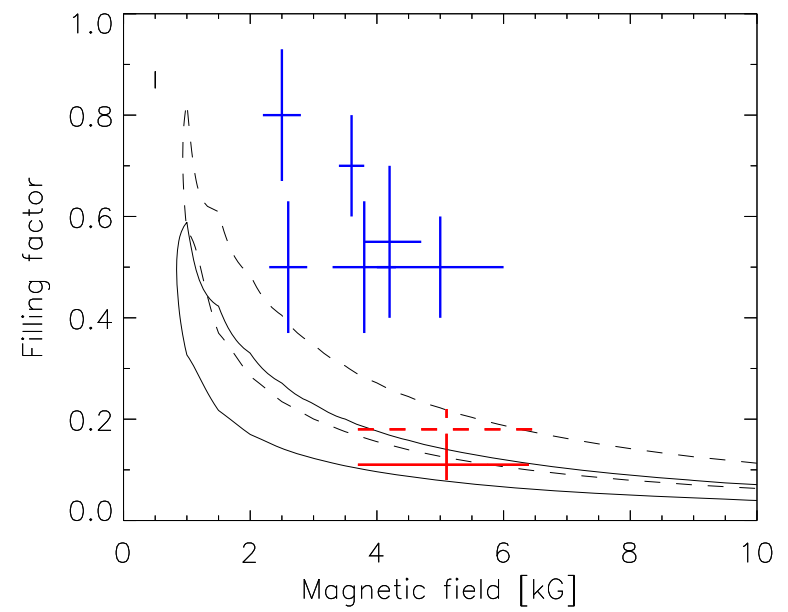

Figure 10. Best-fit magnetic field strengths and filling factors for the LSR $\mathrm{J} 1835$ average Stokes $V / I$ profile shown in Figure 2. The solid-line and dashedline contours include feasible solutions for a longitudinal $\left(\gamma=180^{\circ}\right)$ and inclined $\left(\gamma=130^{\circ}\right)$ fields, respectively. The red solid and dashed crosses mark the best solutions within the two contours, which are compatible with the area of the active region recovered from emission lines. Blue crosses indicate field parameters measured on M dwarfs (Johns-Krull \& Valenti 1996; Berdyugina 2005) within their uncertainties.

$17 \pm 4 \%$. This is a factor of two to three smaller than inferred on early red dwarfs (e.g., EV Lac; Figure 10). Whether this is related to a smaller magnetic flux or a larger field complexity is to be clarified from high-resolution spectropolarimetry and MHD simulations.

Our measurements were carried out one month after a campaign of simultaneous radio and optical observations of LSR J1835 using Jansky Very Large Array (VLA) and LRIS spectrometer at Keck, respectively (Hallinan et al. 2015). Those observations confirmed the correlation of the optical emission with radio bursts. Also, it was suggested that this phenomenon is somewhat similar to auroras in planetary atmospheres bombarded by stellar wind particles. Our detection of the circular polarization signature due to a strong magnetic field near the maximum of the optical emission indicates that radio, optical, and magnetic signals originate from the same region, which extends from the visible surface to higher layers above.

We can obtain indirect estimates of the magnetic field in the radio emission source regions, assuming that they radiate as an ECM. This mechanism implies that radiation is emitted at the electron cyclotron frequency $\nu_{\mathrm{c}} \approx 2.8 \times B \mathrm{MHz}$, where $B$ is the magnetic field strength in Gauss (e.g., Hallinan et al. 2007). We obtain $B$ values along the loops L1 and L2 as shown in Figure 7 (right panels): $1.5-1.6 \mathrm{kG}$ above $\mathrm{H} \gamma, 1.5-1.9 \mathrm{kG}$ within the $\mathrm{H} \gamma$ regions, and $2.5-2.8 \mathrm{kG}$ within the $\mathrm{H} \beta$ regions. These loops are anchored at the magnetic spot with $5.1 \mathrm{kG}$ in the photosphere.

Hallinan et al. (2015) estimated the bursting region to occupy only a few percent of the visible dwarf surface. Our models indicate that the field strength within such a small region could be stronger than $10 \mathrm{kG}$. Whether such a strong field can exist on the brown dwarf surface remains to be clarified. An independent analysis of Stokes parameters within molecular bands, primarily in the $\mathrm{CrH} 860-870 \mathrm{~nm}$ band, which is also in the PBR, supports our detection of the $5 \mathrm{kG}$ field near the phase 0.0 (Kuzmychov et al. 2017). Also, the fact 
that the field is directed from the observer, with the inclination angle of about $130^{\circ}$ agrees with the estimate from the $\mathrm{CrH}$ band of about $115^{\circ}$ near phase 0.0. This further supports the recovered geometry of the magnetic emission loops, which are significantly inclined to the line of sight. Interestingly, the $\mathrm{CrH}$ polarization was marginally seen also at other rotational phases with smaller filling factors, which indicates that magnetic field may be small-scale and ubiquitous.

Different triggers can be considered for the strong and lasting bursts on LSR J1835 and similar brown dwarfs. They do resemble planetary auroras, as was suggested by Hallinan et al. (2015), when the planet magnetosphere is perturbed by the near-planetary environment, e.g., by stellar wind, as in the case of the Earth, or a moon's volcanic activity, as in the case of Jupiter and Io in the solar system. An auroral nature of the bursts on LSR J1835 requires electron beams driven by largescale current systems. Then, the emission will vary with variation of the current system. This is possible if, for example, LSR J1835 would host a planet with a magnetosphere. In light of the recent discovery of the Proxima b around the M5.5 dwarf (Anglada-Escudé et al. 2016) and the M8.5 dwarf TRAPPIST1 system with seven planets on closely packed orbits (Gillon et al. 2016), such "star-planet" interactions could power auroral activity on ultra-cool and brown dwarfs and their planets.

On the other hand, the relatively young age of LSR J1835 of $22 \mathrm{Myr}$ implies that it should still possess some disk matter (Baraffe et al. 2017) and perhaps continue accretion via magnetospheric accretion, as proposed for T Tau stars (Hartmann et al. 1994; Shu et al. 1994). Observations of young clusters and associations suggest that dissipation of disks is less efficient for brown dwarfs than for higher-mass stars (e.g., Riaz et al. 2012; Riaz \& Kennedy 2014; Downes et al. 2015). In low-mass stars $\left(0.1-1 M_{\odot}\right)$, the transition from primordial disks to debris disks occur at about $10 \mathrm{Myr}$, and debris disks peak at the age of 10-30 Myr. In contrast, brown dwarf disks are still in the primordial stage at $10 \mathrm{Myr}$, and their debris disks seem to largely disappear by the age of 40-50 Myr (Riaz \& Kennedy 2014), with rare exceptions (Boucher et al. 2016). Thus, it is possible that LSR J1835 still has a disk. Interestingly, the observed IR spectral energy distribution (SED) of the dwarf shows an excess that requires a significantly cooler atmosphere with $T_{\text {eff }}=2200 \mathrm{~K}$ and warm dust (BT-Settl models) than deduced from spectral lines (Kuzmychov et al. 2017). Whether this is a consequence of the dust in the dwarf atmosphere or in a disk or can be due to the presence of cool starspots (or a combination of the three) has yet to be clarified. In particular, broadband polarimetry can help distinguish between dust in a disk and in the atmosphere, while high-resolution spectropolarimetry is needed to improve parameters of starspots.

The presence of a disk may facilitate large-scale magnetic reconnections between the dwarf magnetosphere and the disk, similar to T Tau-type stars, as mentioned above (see also Zhu et al. 2009). In this scenario, the strong stellar magnetic field truncates the inner edge of the disk at the distance where viscous ram pressure equalizes the magnetic pressure. In preMS stars, this magnetospheric radius may reach 5-10 stellar radii. Inside this radius, the matter sporadically flows along magnetic field lines until it impacts the stellar surface and causes bursts (flares). Magnetic loops on brown dwarfs (as observed in the radio) may extend one to three dwarf radii from the surface (Lynch et al. 2015). Thus, it is possible that radiobursting ultra-cool dwarfs are in the T Tau phase of magnetospheric accretion. It would be necessary to verify whether they possess $\mathrm{kG}$-strong magnetic fields and are of young enough age, similar to LSR J1835.

In addition, larger filling factors for the equipartition field of $5 \mathrm{kG}$ (i.e., the most probable field strength for given plasma conditions) require wide-spread mixed-polarity magnetic fields similar to what is observed on the Sun as a small-scale, intergranular network field. This field constantly emerges from the turbulent interior, as is observed on the Sun and is predicted for fully convective ultra-cool dwarfs to be dominated by a highly entangled field at the equipartition level throughout the atmosphere. Then, this complex, rather strong field can also trigger explosive events. Such a complex field would frequently rearrange itself through reconnections leading to flares, aurora-like emission, and radio pulses, modulated by fast rotation of brown dwarfs. A combination of such surface magnetic field evolution with possible dwarf-disk or dwarfplanet interactions would be an exciting opportunity to learn about early stages of the planet formation in the presence of large-scale magnetosphere interactions.

\section{Summary and Conclusions}

We have measured near-infrared polarized (Stokes IQUV) and optical emission spectra of the active M8.5 young brown dwarf LSR J1835+3259 using the LRISp spectropolarimeter at the Keck telescope during two consecutive nights on 2012 August 22-23. Measurements were made at several aspect angles during two rotational periods separated by seven periods. The $5.1 \mathrm{kG}$ magnetic field was detected as a Zeeman signature in the near-infrared $\mathrm{Na}$ I $819 \mathrm{~nm}$ lines during the first night when an active region radiating non-thermal optical emission faced the Earth (near phase 0.0). The magnetic field is found to cover at least $11 \%$ of the dwarf visible hemisphere.

By employing the sensitivity of the NIR Na I and TiO lines in the $819 \mathrm{~nm}$ region to the temperature and gravity, we have determined atmospheric parameters $T_{\text {eff }}=2800 \pm 30 \mathrm{~K}$ and $\log g=4.50 \pm 0.05$ with high accuracy. A comparison with evolutionary models leads us to the conclusion that LSR J1835 +3259 is a young brown dwarf with the mass $M=55 \pm 4 M_{\mathrm{J}}$ and age $t=22 \pm 4 \mathrm{Myr}$. Thus, its prominent magnetic activity is probably related to its young age.

We have inferred the emitting region topology using optical emission line profile inversions. We have found that model line profiles corresponding to a decaying stage of an energetic flare on red dwarfs are well suited for such inversions. The emission maps obtained from inversions indicate the presence of hot plasma loops of at least $7000 \mathrm{~K}$ with a vertical stratification of the emission sources. In particular, it was found that higherexcitation emission peaks at earlier phases than the lower excitation emission. As the dwarf rotates with the $2.84 \mathrm{hr}$ period, these loops rotate in and out of view and cause the emission modulation observed as periodic bursts. The emission near the phase 0.0 was the strongest on 2012 August 22, and decayed by 2012 August 23, i.e., on the timescale of several rotation periods, making such periodic bursts transient. At the same time, a second emission region was emerging at almost opposite longitude (phase 0.6) toward the end of our observing campaign. 
The region near the phase 0.0 was also active (in optical and radio emission) a month before our measurements (Hallinan et al. 2015). Hence, the life time of this active region is at least a month, and at least two transient flare-like events occurred in this region. It was also puzzling that radio pulses were arriving to Earth at somewhat earlier phases than $\mathrm{H} \alpha$ emission. Our emission maps interpreted as projections of extended corotating loops indicate that the high-frequency radio emission sources appear co-spatial with high-excitation optical emission (perhaps due to projection).

The $5 \mathrm{kG}$ magnetic field was detected at the base of the emission loops near the phase 0.0. This is the first time that we can quantitatively associate radio and optical bursts with a strong, $5 \mathrm{kG}$ surface magnetic field required by the ECM instability mechanism. When assuming the ECM mechanism, the magnetic field strength along the loops seems reduce from $5 \mathrm{kG}$ at the base to $1.5 \mathrm{kG}$ at the highest observed location, which can be as high as three dwarf radii from the surface (Lynch et al. 2015). Whether this magnetic region is associated with the global magnetic field of the dwarfs is an interesting question. A longer series of polarimetric observations is needed to verify this. Also, high-resolution full-Stokes spectropolarimetry can help to map magnetic field and emission regions on this dwarf with a better spatial resolution.

We conclude that the activity on LSR J1835+3259 and possibly other ultra-cool and brown dwarfs with non-thermal radio and optical emission bursts is associated with a strong magnetosphere driven by a few $\mathrm{kG}$ surface magnetic fields, similar to what is observed on active red dwarfs. An interaction of a large-scale magnetic field (long-lived active regions or magnetic poles) with small-scale, entangled, widespread, and rapidly evolving magnetic fields and possibly with a magnetized disk or a planet (to be confirmed) may lead to frequent reconnection events and trigger optical and radio bursts. Since this is the coolest known dwarf with such a strong magnetic field, our result also provides a unique constraint for simulations of magnetic fields in fully convective ultra-cool dwarfs (e.g., Dobler et al. 2006; Browning 2008; Yadav et al. 2015). Considering that hot Jupiter-like exoplanets are of similar temperatures as brown dwarfs, our result brings us closer to studying the magnetism of hot Jupiters.

This work was supported by the ERC Advanced Grant HotMol (http://www.hotmol.eu) ERC-2011-AdG-291659. Based on observations made with the Keck Telescope, Mauna Kea, Hawaii. We thank the Keck staff, support astronomers and, in particular, Dr. Bob Goodrich and Dr. Hien Tran for their support. S.V.B. acknowledges the support from the NASA Astrobiology Institute and the Institute for Astronomy, University of Hawaii, for the hospitality and allocation of observing time at the Keck telescope. The authors wish to recognize and acknowledge the very significant cultural role and reverence that the summit of Mauna Kea has always had within the indigenous Hawaiian community. we are most fortunate to have the opportunity to conduct observations from this mountain. We thank an anonymous referee for a constructive and helpful report.

\section{ORCID iDs}

O. Kuzmychov (iD https://orcid.org/0000-0001-5186-5547

S. L. Hawley (iD https://orcid.org/0000-0002-6629-4182

\section{References}

Afram, N., Berdyugina, S. V., Fluri, D. M., et al. 2007, A\&A, 473, L1 Afram, N., Berdyugina, S. V., Fluri, D. M., Solanki, S. K., \& Lagg, A. 2008, A\&A, 482, 387

Allard, F., Homeier, D., Freytag, B., \& Sharp, C. M. 2012, in EAS Publ. Ser. 57, Low-Mass Stars and the Transition Stars/Brown Dwarfs, EES2011, ed. C. Reylé, C. Charbonnel, \& M. Schultheis (Les Ulis: EDP Sciences), 3

Allard, F., Hauschildt, P. H., Alexander, D. R., Tamanai, A., \& Schweitzer, A. 2001, ApJ, 556, 357

Anglada-Escudé, G., Amado, P. J., Barnes, J., et al. 2016, Natur, 536, 437

Antonova, A., Doyle, J. G., Hallinan, G., Golden, A., \& Koen, C. 2007, A\&A, 472, 257

Baraffe, I., Elbakyan, V. G., Vorobyov, E. I., \& Chabrier, G. 2017, A\&A, 597, A19

Bell, R. A., Edvardsson, B., \& Gustafsson, B. 1985, MNRAS, 212, 497

Berdyugina, S. V. 1998, A\&A, 338, 97

Berdyugina, S. V. 2005, LRSP, 2, 8

Berdyugina, S. V., \& Solanki, S. K. 2002, A\&A, 385, 701

Berdyugina, S. V., Frutiger, C., Solanki, S. K., \& Livingston, W. 2000, A\&A, 364, L101

Berdyugina, S. V., Solanki, S. K., \& Frutiger, C. 2003, A\&A, 412, 513

Berdyugina, S. V., Braun, P. A., Fluri, D. M., \& Solanki, S. K. 2005, A\&A, 444, 947

Berdyugina, S. V., Fluri, D. M., Ramelli, R., et al. 2006a, ApJL, 649, L49

Berdyugina, S. V., Petit, P., Fluri, D. M., Afram, N., \& Arnaud, J. 2006b, in ASP Conf. Ser. Solar Polarization 4, ed. R. Casini \& B. W. Lites (San Francisco, CA: ASP), 381

Berger, E. 2006, AJ, 648, 629

Berger, E., Rutledge, R. E., Reid, I. N., et al. 2005, ApJ, 627, 960

Berger, E., Ball, S., Becker, K. M., et al. 2001, Natur, 410, 338

Boucher, A., Lafreniére, D., Gagné, J., et al. 2016, ApJ, 832, 50

Browning, M. K. 2008, ApJ, 676, 1262

Burrows, A., Marley, M., Hubbard, W. B., et al. 1997, ApJ, 491, 856

Davis, S. P., Phillips, J. G., \& Littleton, J. E. 1986, ApJ, 309, 449

Deshpande, R., Martin, E. L., Montgomery, M. M., et al. 2012, AJ, 144, 99

Dobler, W., Stix, M., \& Brandenburg, A. 2006, ApJ, 638, 336

Downes, J. J., Román-Zúñiga, C., Ballesteros-Paredes, J., et al. 2015, MNRAS, 450,3490

Frutiger, C., Solanki, S. K., Fligge, M., \& Bruls, J. H. M. J. 2000, A\&A, 358, 1109

Gillon, M., Jehin, E., Lederer, S. M., et al. 2016, Natur, 533, 221

Grosso, N., Briggs, K. R., Güdel, M., et al. 2007, A\&A, 468, 391

Gustafsson, B., Edvardsson, B., Eriksson, K., et al. 2008, A\&A, 486 951

Hallinan, G., Antonova, A., Doyle, J. G., et al. 2006, ApJ, 653, 690

Hallinan, G., Bourke, S., Lane, C., et al. 2007, ApJL, 663, L25

Hallinan, G., Littlefair, S., Cotter, G., et al. 2015, Natur, 523, 568

Harding, L. K., Hallinan, G., Boyle, R. P., et al. 2013, ApJ, 779, 101

Harrington, D. M., Berdyugina, S. V., Kuzmychov, O., \& Kuhn, J. R. 2015, PASP, 127, 757

Hartmann, L., Hewett, R., \& Calvet, N. 1994, ApJ, 426, 669

Johns-Krull, C., \& Valenti, J. 1996, ApJL, 459, L95

Johns-Krull, C., \& Valenti, J. 2000, in ASP Conf. Ser. 198, Stellar Clusters and Associations: Convection, Rotation, and Dynamos, ed. R. Pallavicini, G. Micela, \& S. Sciortino (San Francisco, CA: ASP), 371

Kowalski, A. F., Hawley, S. L., Carlsson, M., et al. 2015, SoPh, 290, 3487

Kowalski, A. F., Allred, J. C., Uitenbroek, H., et al. 2017, ApJ, 837 125

Kurucz, R. 1993, Kurucz CD-ROM No. 1 (Cambridge, MA: Smithsonian Astrophysical Observatory)

Kuzmychov, O., \& Berdyugina, S. V. 2013, A\&A, 558, A120

Kuzmychov, O., Berdyugina, S. V., \& Harrington, D. M. 2017, ApJ, 847, 60

Kuznetsov, A. A., Doyle, J. G., Yu, S., et al. 2012, ApJ, 746, 99

Luhman, K. L. 2012, ARA\&A, 50, 65

Lynch, C., Mutel, R. L., \& Güdel, M. 2015, ApJ, 802, 106

Mohanty, S., \& Basri, G. 2003, ApJ, 583, 451

Neuhäuser, R., Briceño, C., Comerón, F., et al. 1999, A\&A, 343, 883

Plez, B. 1998, A\&A, 337, 495

Preibisch, T., McCaughrean, M. J., Grosso, N., et al. 2005, ApJS, 160 582

Press, W. H., Teukolsky, S. A., Vetterling, W. T., \& Flannery, B. P. 2007, Numerical Recipes: The Art of Scientific Computing (Cambridge: Cambridge Univ. Press)

Reiners, A., \& Basri, G. 2010, ApJ, 710, 924 
Riaz, B., \& Kennedy, G. M. 2014, MNRAS, 442, 3065

Riaz, B., Lodieu, N., Goodwin, S., Stamatellos, D., \& Thompson, M. 2012, MNRAS, 420, 2497

Schiavon, R. P., Barbuy, B., \& Singh, P. D. 1997, ApJ, 484, 499

Schmidt, S. J., Cruz, K. L., Bongiorno, B. J., Liebert, J., \& Reid, I. N. 2007, AJ, 133, 2258

Shu, F., Najita, J., Ostriker, E., et al. 1994, ApJ, 429, 781
Sobelman, I. I. 1992, Atomic Spectra and Radiative Transitions (2nd ed.; Berlin: Springer)

Yadav, R. K., Christensen, U. R., Morin, J., et al. 2015, ApJL, 813 L31

Zapatero Osorio, M. R., Martín, E. L., Béjar, V. J. S., et al. 2007, ApJ, 666,1205

Zhu, Z., Hartmann, L., \& Gammie, C. 2009, ApJ, 694, 1045 